\title{
Rethinking psychiatry with OMICS science in the age of personalized P5 medicine: ready for psychiatome?
}

\author{
Nicola Luigi Bragazzi ${ }^{1,2}$
}

\begin{abstract}
The Diagnostic and Statistical Manual of Mental Disorders (DSM) is universally acknowledged as the prominent reference textbook for the diagnosis and assessment of psychiatric diseases. However, since the publication of its first version in 1952, controversies have been raised concerning its reliability and validity and the need for other novel clinical tools has emerged. Currently the DSM is in its fourth edition and a new fifth edition is expected for release in 2013, in an intense intellectual debate and in a call for new proposals.

Since 1952, psychiatry has undergone many changes and is emerging as unique field in the medical area in which a novel approach is being demanded for properly treating patients: not the classical "one-size-fits-all" approach, but a more targeted and tailored diagnosis and therapeutics, taking into account the complex interactions among genes and their products, environment, culture and the psychological apparatus of the subject.

OMICS sciences, being based on high-throughput technologies, are systems biology related fields (like genomics, proteomics, transcriptomics and so on). In the frame of the P5 medicine (personalized, participatory, predictive, preventive, psycho-cognitive), they could establish links between psychiatric diseases, which are disorders with a final common symptomatology with vastly heterogeneous biological, environmental and sociological underpinnings, and by understanding the psychiatric diseases beyond their classic symptomatic or syndromal definitions using OMICS research, one can have a broader picture and unprecedented links and reclassification of psychiatric nosology. Importantly, by understanding the basis of heterogeneity in diseases through OMICS research, one could also personalize treatment of psychiatric illnesses.

In this manuscript, we discuss a gap in the current psychiatric research, namely the missing logical link among OMICS, personalized medicine and reclassification of diseases. Moreover, we explore the importance of incorporating OMICSbased quantitative dimensional criteria, besides the classical qualitative and categorical approach.
\end{abstract}

Keywords: Biological psychiatry, Systems biology, Network medicine, Bioinformatics, New psychiatry, Personalized medicine, DSM-V, OMICS

\section{Italian abstract}

Please see Additional file 1 for the translation of the abstract into Italian.

\section{Introduction}

Traditionally, psychiatric diseases have been considered as a cluster of symptoms (syndromes) and psychopathology

\footnotetext{
Correspondence: nicola@genethics.ethicsoft.it

${ }^{1}$ Department of Health Sciences (DISSAL), School of Public Health, University of Genoa, Via Pastore 1, 16132, Genoa, Italy

2DINOGMI, Department of Neuroscience, Rehabilitation, Ophthalmology, Genetics, Maternal and Child Health, Section of Psychiatry, University of Genoa, Genoa, Italy
}

has been the gold standard to make diagnosis. However psychiatric diseases are complex, multifaceted and multifactorial pathologies, characterized by high heterogeneity and variance and therefore classical methods have proven to be too simple or not completely adequate to capture this complexity [1].

The categorical approach, in fact, suffers from some drawback, like circular reasoning and ambiguity [2], in considering diseases such as static categories and discrete ontologies, separate one from the others. Moreover, it is very puzzling where to set the boundary between the "normal" (health status) and the "abnormal" (the disease) 
and this has not only academic and nosological issues, but above all social and political concerns [2].

Another disadvantage of using the categorical approach alone is the nosological overlap: under the same clinical umbrella, different diseases with different prognosis can co-exist. Categorical approach, being qualitative, should be complemented with a more fine-grained diagnostic tool. On the other hand, molecular classification can really help and improve the classical nosological taxonomy and thus ameliorate the outcome of patient management and care.

This aspect of integrated psychological and biological assessment, that is to say both quantitative and qualitative, categorical and dimensional, is to be stressed within the frame of personalized medicine (P5) and targeted therapeutics, which recently emerged as promising and exciting trends. P5 medicine (i.e., predictive, preventive, personalized, participatory and psycho-cognitive) [3-5] entails the addition of the psycho-cognitive domain as a conceptual evolution of $\mathrm{P} 4$ medicine, introduced by the molecular oncologist Leroy Hood [6]. P5 medicine, as advocated by Gorini, Pravettoni and Ozdemir, has abandoned the model of paternalism that characterized the $20^{\text {th }}$ century physician-patient interaction. Instead P5 is participatory and makes use of models and equations to predict patient's future health status in order to adopt the best strategy available (being predictive); is preventive in being proactive and not merely reactive; and is psycho-cognitive in that it adopts an integrated model in which psychological health is seen as a fundamental aspect of personal well-being. Psychology plays different roles in medicine: from the compliance and adherence to treatment, to the access of the treatment itself (traditional versus alternative or complementary) and ultimately to the therapeutic alliance of the broad meaning. The concept of disease is thus shifting from an atheoretical, context-free, "Platonic" model, to an approach that instead is more focused on patient's characteristics and needs [5].

While P5 medicine is biology-driven (personalized, predictive, preventive), it is also psychologically and ethically engaged (psychocognive, participatory). Therefore, it is really patient-centered.

Thus, psychiatry is emerging as something of a unique medical field in which a novel approach is demanded and needed for properly treating patients through a more targeted and tailored diagnosis and therapy [1-14], taking into account the complex interactions among genes and their products, environment, and culture (as shown in Figure 1).

For this reason, both molecular (studied through novel biotechnologies such as gene microarrays and/or protein arrays) and psychodynamic aspects must be considered in order to fulfill the promises and true potential of a personalized psychiatry $[15,16]$.
Moreover, since its very beginning, psychiatry has always had societal, ethical and political implications. The negative stereotypes, social stigma, and host of discriminating policies and discredit have hindered the process of psychiatric rehabilitation. P5 medicine, being multi-scalar and multi-dimensional, include the patient as an actor in the therapeutic process and incorporates "open knowledge production systems" in a truly holistic approach, may well capture the original concept of the individual person to acknowledge the dignity of the patient in the formulation and application of acts of care [17].

\section{From classical qualitative and categorical approaches to dimensional and integrated approaches}

In this first section, we provide a brief overview of the historicity of the main approaches that have been proposed for a unitary psychiatric taxonomy, emphasizing the shift from a categorical qualitative approach, to a more refined dimension methodology.

\section{DSM/ICD model}

The Diagnostic and Statistic Manual of Mental Disorders (DSM) was originally developed in 1952, upon the publication of the DSM-I. The DSM represents the first tool developed for ad hoc psychiatric diagnosis, even though its sources were implemented for epidemiological and social policy purposes, and not (at least initially) for the sake of diagnosis and treatment.

It is important to emphasize that DSM is a work in progress, and has undergone many changes through its evolution, shifting from a philosophical and phenomenological approach, to one that is more qualitative and psychometric, incorporating neo-Kraepelinian orientation (for an overview, the reader is referred to [2]).

The so-called Feighner criteria (and their expanded version, the Research Diagnostic Criteria, RDC, of the National Institute of Mental Health, NIMH, Psychobiology of Depression Collaborative Study, in 1978) have been acknowledged as the foundation of the version of DSM-III, which also incorporated the Robins-Guze criteria (i.e., describing five phases of psychiatric diagnostic formulation, namely clinical description, laboratory analysis, exclusion of other disorders, follow-up and family study) [18].

This same methodology has informed the International Statistical Classification of Diseases and Related Health Problems (ICD), developed by World Health Organization (WHO), while the Psycho-dynamic Diagnostic Manual (PDM) remains the psychoanalytical counterpart [19].

Although it was greatly improved throughout its different versions, problems persist in the DSM. Among the main limitations are the arbitrary threshold for diagnosis, the lack of quantitative criteria, potential abuse and/or misuse of the not otherwise specified (NOS) 


\section{ENVIRONMENT}

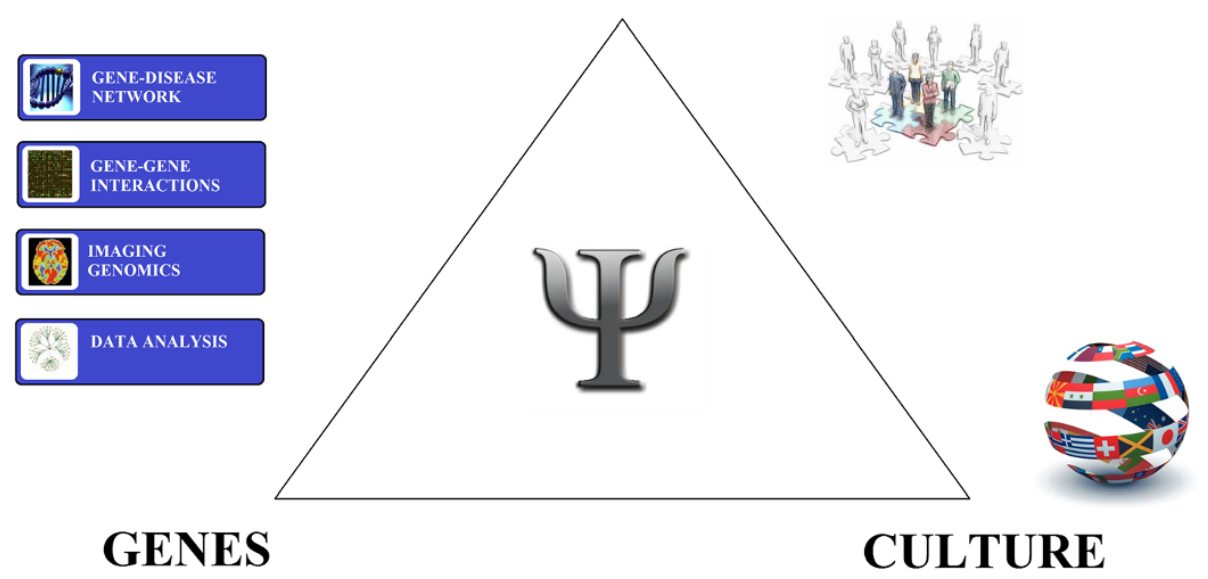

Figure 1 Triangle showing interactions among human genome, environment and culture in the pathogenesis of psychiatric diseases.

diagnostic category and overlap resulting from multiaxial structure (i.e., the "co-morbidity issue").

As well, some empirical categories have disappeared from one version to the next, (e.g., homosexuality and some personality disorders), while others have been introduced (e.g., post-traumatic stress disorder, (PTSD)). Such categorical variation strongly suggests the need for more rigorous and evidence-based definition of diseases, as directly relevant to clinical diagnoses and treatment(s).

\section{Biopsychosocial model}

The biopsychosocial model was first formally proposed by the psychiatrist George Engel [20] and has been incorporated - to greater or lesser extent - in the subsequent versions of the DSM. The model introduces distinctions within and between biological, psychological and social components, and advocates a more holistic orientation to disease, illness and medical care. However, post-Engelian interpretations of the biopsychosocial approach have tended to be somewhat obtuse [21-23]. Eric Kandel and Antonio Damasio have noted the linked and interwoven nature of a true biopsychosocial approach, such that psychiatric care does not belong to a separate and remote "psychological sphere" but also reflects and entails biological effects. In the same way, social components cannot be considered as completely distinct from biological and psychological domains.

\section{Meta-structure theory}

Instead of a classification based on clinical presentation (as in DSM-IV, ICD10, and/or PDM), meta-structural taxonomy was proposed by Andrews and collaborators [24] as based on risk factors and intended as a more parsimonious classification. The proposed clusters are: neurocognitive disorders (cluster 1), neuro-developmental disorders (cluster 2), psychoses (cluster 3), emotional disorders (cluster 4), externalizing disorders (cluster 5) [24]. The proposed axes are only two and currently two variants exist.

Yet, this approach suffers from many of the same limitations as the previously described orientations.

\section{From categorical approaches to dimensional taxonomies:} Endophenotype theory

An endophenotypic approach represensts a considerable step further and it is intriguing to note that this model although proposed for the psychiatric diseases, also may be extended to other diseases (e.g., diabetes, cancer, metabolic pathologies, etc.).

The endophenotype makes use of biological markers, described using the Buchsbaum criteria (i.e., a biomarker or a biological trait as a measurable indicator of a disease, which may be or may be not casual) and the Gottesman-Gould criteria for diagnosing an endophenotypic trait, which are that 1) the endophenotype is associated with illness in the population; 2) the endophenotype is heritable; 3) the endophenotype is disease state-independent; and 4) endophenotype and illness co-segregate within families [25].

This is fundamental to the suggested changes we propose to inform the use of the new DSM-V.

\section{Insights from systems biology, neuroinformatics and OMICS sciences}

We posit that the traditional conception of "homeostasis" and the difficulty of distinguishing "normal" and an "abnormal" status can be compensated - if not resolved to great extent - by the idea of a personalized inner dynamical equilibrium that exist among the genetic and biological components, developed and expressed psychological resources traits and expressions, and the environment. Baselines and 
threshold values for biological (and, by effect psycho-social ) system sensitivities and responses vary from person to person, as well in the same person throughout the lifespan. Thus the shift from a healthy status to a pathological status is often not a binary condition, but rather reflects a continuum, wherein disease emerges as a transformation of the properties of system(s) that have gradually become less adaptive [26-28].

Not merely focusing on single pieces of the proverbial puzzle, but instead taking a more comprehensive view of the picture that entails the systems involved, with and through approaches from systems biology, neuroinformatics and OMICS-based sciences to be implemented in psychiatric assessment and treatment [26].

According to etymology, OMICS is derived from the Sanskrit OM, which means "completeness and fullness", and thus, a holistic, systems-oriented approach [29], which may be well-positioned to fill the gap between the need for a rigorous and rational psychiatry and the need for a personalized medicine.

\section{The "Psychiatome"}

The interplay of genomics, proteomics, transcriptomics, toponomics, metabolomics and neuroimaging is emerging

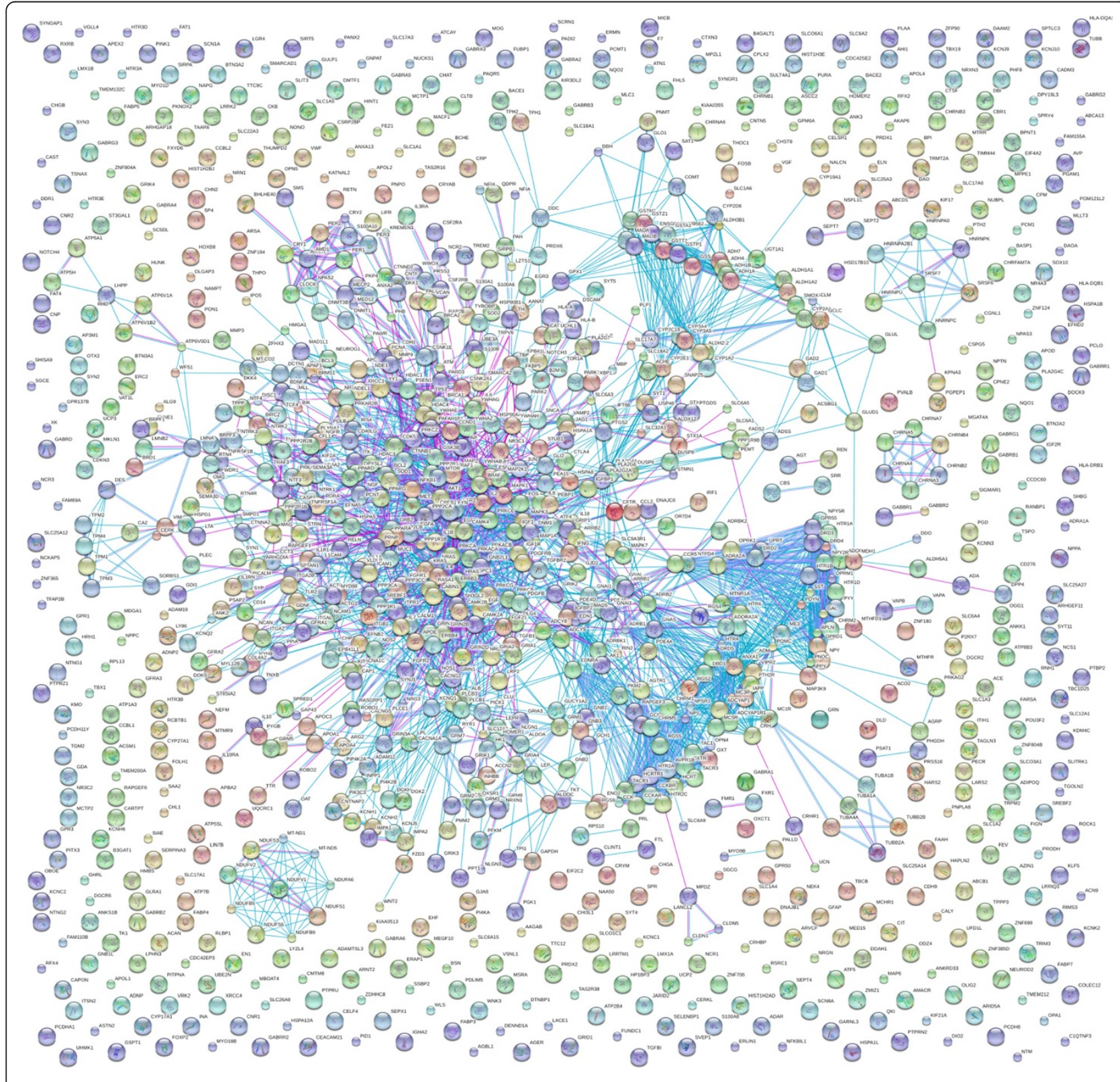

Figure 2 Gene-gene connections of all genes involved in psychiatric diseases. Edges between nodes represent the known molecular interactions. 


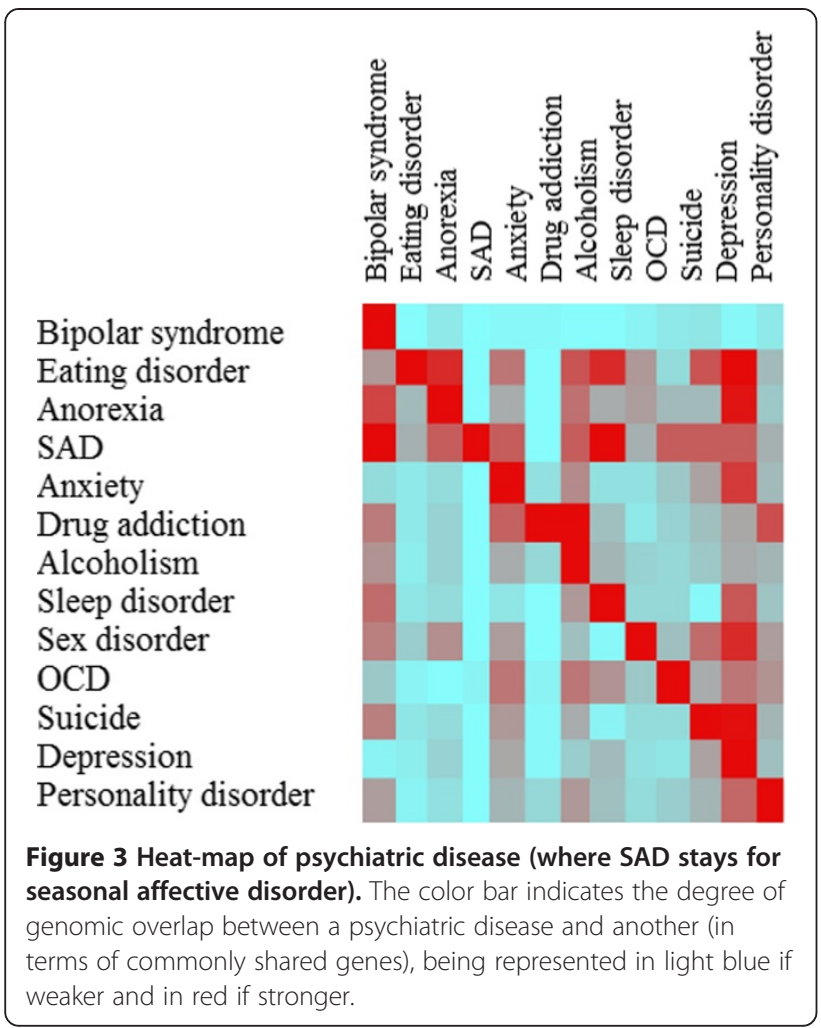

as powerful tool to analyze psychiatric disorders and providing patients personalized care. Barabási introduced the concept of "diseasome" [30] in the context of network medicine. Herein, we investigate the putative relationships among and between biological and environmental factors in psychiatric diseases, in what we call "psychiatome", inspired by the concept of "diseasome".

Our approach is to assess the shared genes (via mining through genetic databanks), shared networks (using inferred proteomics data) and shared networks of anatomical regions (i.e. toponomics, exploiting the PubBrain tool) in such a way as to develop a unique picture of salient inter-relationships that may subserve or be reflected by psychiatric disorders.

In attempting re-construct the complex genetic architecture of the psychiatric disorders (as multi-factorial diseases), we take into account whole-genome association (WGA) studies, copy-numer variant $(C N V)$, pathwaysbased analysis $(P B A)$, micro-arrays-based studies, single nucleotide polymorphisms (SNPS) studies, quantitative trait, loci and allelic heterogeneity studies.

Other complex diseases (e.g., heart diseases, diabetes, asthma, hypertension) are in fact characterized by the involvement of different genes. The possible connections among different genes involved in our proposed psychiatome schema are shown in Figure 2, and the heat-map matrix depicting relationships among the different psychiatric diseases is represented in Figure 3, with their respective gene onthologic (GO) functional enrichment in Figure 4. Delineating common shared-genes and genomicsrelationships among and between psychiatric diseases can be useful in shaping the concept of "spectrum" disorder (as merely a static binary notion of "disease") and preparation for use of the new DSM-V in practice [31].

But a truly personalized psychiatry is a broader concept than simply of pharmacogenomics, genotyping or other molecular investigations: it also includes the cultural and spiritual beliefs and practices of the patient [32]. This is particularly true also because from an evolutionary point of view, culture and genes have co-evolved such that culture can influence human genome [33-35], just as the expression of traits and actions arising from the genome can affect culture [36], as pictorially shown in Figure 1.

\section{Discussion}

In this light, we believe that there is urgent need for a more comprehensive personalized psychiatric medicine [37]. Moving from the well-known epidemiological

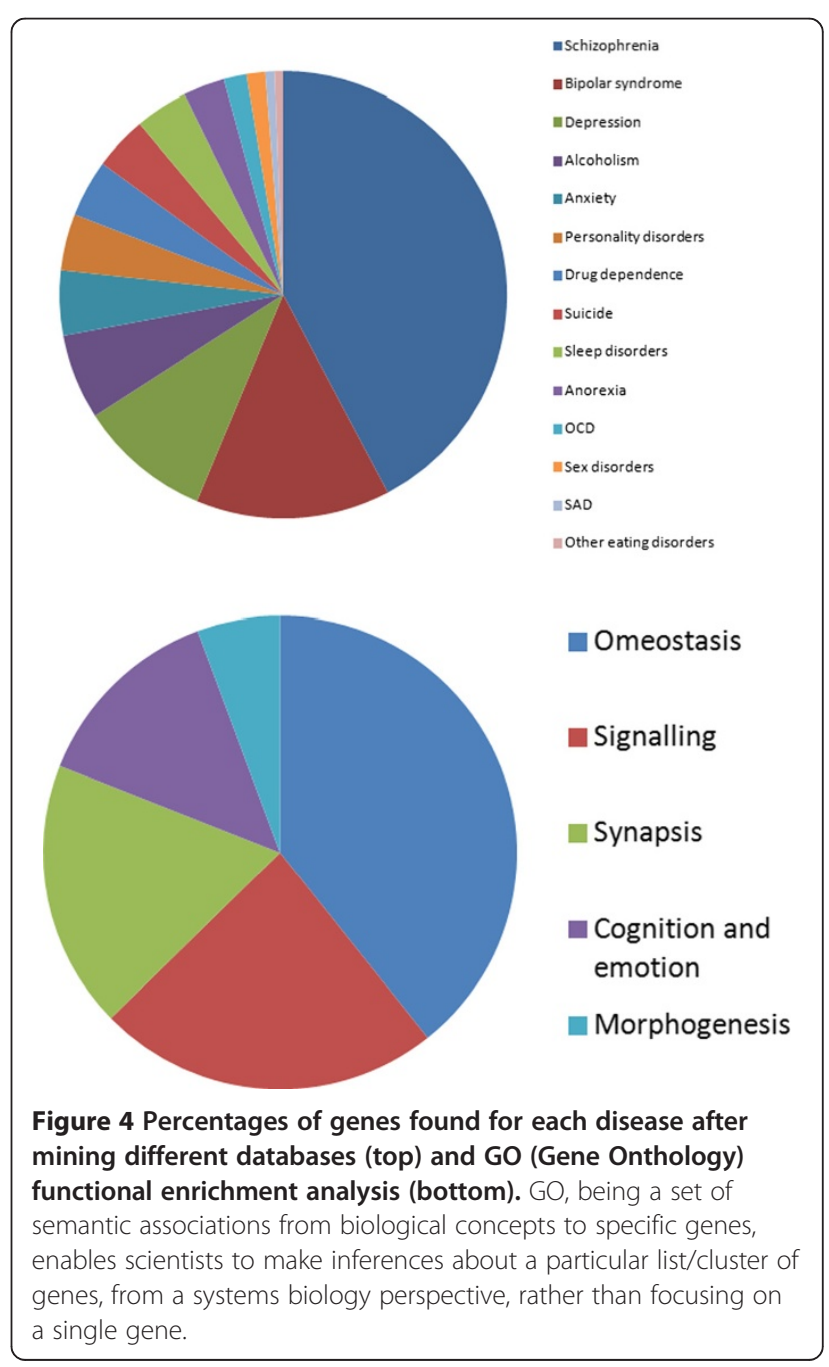


triangle (Figure 1), we have postulated the necessity of considering psychiatric disease from multiple points of view, given that psychiatric disorders are the result of complex gene-environment-culture interactions and we have over-viewed some tools doctors can exploit.

Psychiatric diseases tend to be interrelated (Figures 2 and 3), and exert distinct molecular signatures, and behavioral markers [38].

Molecular and genomic algorithms [39-41], nanobiotechnologies [42-45], OMICS-derived data and clinical tests and questionnaires [46] can all be useful to unravel the ambiguities of psychiatric pathogenesis if - and only if - combined and integrated.

Classical qualitative approaches have limitations on both theoretical and pragmatic grounds. Considering a more dimensional methodology, could overcome many of these limitations and thereby be instrumental to improving psychiatric diagnoses and treatments. OMICS sciences, based on high-throughput technologies, are systems biology related fields, which in a framework of P5 medicine could help to establish critical links between psychiatric diseases, (as disorders with a final common symptomatology with vastly heterogeneous biological, environmental and sociological underpinnings), and could thereby afford an understanding of psychiatric disease beyond classic symptomatic or syndromal definitions, perhaps leading to better interpretation and use of psychiatric nosology. Perhaps most importantly, by fostering such understanding through OMICS research, could also enable a meaningfully personalization of psychiatric care.

\section{Conclusion}

In this paper, we offer but an early and limited view to the potential benefits of OMIC-science in, and for psychiatry. Further studies must be undertaken to better characterize the relationships between genes, culture, and environments, but we hold that a P5-based psychiatry can uphold these tasks.

We have addressed what we believe to be a gap in current psychiatric research and practice, namely, the missing link between OMICS, reclassification of psychiatric disorder and personalized medicine. In posing the importance of incorporating OMICS-based quantitative dimensional criteria, (supplemental to the classical qualitative and categorical approaches), we assert that steps toward a new psychiatry should include:

1) incorporating OMICS-based data to diagnostic criteria, taking biological and OMICS-derived markers not as external validators, but as intrinsic components of assessment;

2) shift emphasis from reactive, post-hoc assessments, to more quantitative diagnostic and prognostically predictive approaches;
3) provide bio-psychosocial (and culture-spiritual) personalized diagnoses and treatments.

Perhaps the "psychiatome" will provide an adequate translational framework for both psychiatric research and practice, being holistic and broad, rather than narrow and simplistic, even though this promising paradigm at present is still at an early stage of its development and implementation.

\section{Additional file}

\section{Additional file 1: Abstract translated into Italian.}

\section{Competing interest}

The author declares that they have no competing interests.

Received: 2 May 2012 Accepted: 20 June 2013

Published: 12 July 2013

\section{References}

1. Patil T, Giordano J: On the ontological assumptions of the medical model of psychiatry: philosophical considerations and pragmatic tasks. Philos Ethics Humanit Med 2010, 5:3.

2. Kawa S, Giordano J: A brief historicity of the Diagnostic and Statistical Manual of Mental Disorders: issues and implications for the future of psychiatric canon and practice. Philos Ethics Humanit Med 2012, 7:2.

3. Gorini A, Pravettoni G: P5 medicine: a plus for a personalized approach to oncology. Nat Rev Clin Oncol 2011, 8(7):444.

4. Pravettoni G, Gorini A: P5 cancer medicine approach: why personalized medicine cannot ignore psychology. J Eval Clin Pract 2011, 17:594-6.

5. Ozdemir $\vee$, Knoppers BM: One size does not fit all: toward "upstream ethics"? Am J Bioeth 2010, 10(6):42-4.

6. Hood L, Friend SH: Predictive, personalized, preventive, participatory (P4) cancer medicine. Nat Rev Clin Oncol March, 8:184-187.

7. Broich K, Möller HJ: Pharmacogenetics, pharmacogenomics and personalized psychiatry: are we there yet? Eur Arch Psychiatry Clin Neurosci 2008, 258(Suppl. 1):1-2.

8. Möller HJ, Rujescu D: Pharmacogenetics - genomics and personalized psychiatry. Eur Psychiatry 2010, 25:291-293.

9. De Leon J: The future (or lack of future) of personalized prescription in psychiatry. Pharmacol Res 2009, 59(2):81-89.

10. Gurwitz D, Weizman A: Personalized psychiatry: a realistic goal. Pharmacogenomics 2004, 5(2):213-7.

11. Ozdemir V, Basile V, Masellis M, Muglia P, Kennedy JL: Pharmacogenomics and personalized therapeutics in psychiatry. In Neuropsychopharmacology: The fifth generation of progress American College of

Neuropsychopharmacology. Edited by Davis KL, Charney D, Coyle JT, Nemeroff C. New York: Lippincott Williams \& Wilkins; 2002:495-506.

12. Filakovi P, Petek A: Personalized pharmacotherapy in psychiatry. Psychiatr Danub 2009, 21(3):341-346.

13. Müller-Spahn F: Individualized preventive psychiatry: syndrome and vulnerability diagnostics. Eur Arch Psychiatry Clin Neurosci 2008, 258(suppl 5):92-97.

14. De Leon J: AmpliChip CYP450 test: personalized medicine has arrived in psychiatry. Expert Rev Mol Diagn 2006, 6:277-286.

15. Stahl SM: Personalized medicine, pharmacogenomics, and the practice of psychiatry: on the threshold of predictive therapeutics in psychopharmacology? CNS Spectrum 2008, 13:115-118.

16. Jain KK: Personalized medicine. Curr Opin Mol Ther 2002, 4:548-558.

17. Ozdemir V, Fisher E, Dove ES, Burton H, Wright GE, Masellis M, Warnich L: End of the beginning and public health pharmacogenomics: knowledge in 'Mode 2' and P5 medicine. Curr Pharmacogenomics Person Med 2012, 10(1):1-6.

18. Kendler KS, Muñoz RA, Murphy G: The development of the Feighner criteria: a historical perspective. Am J Psychiatry 2009, 167:134-142. 
19. Wallerstein RS: The Psychodynamic Diagnostic Manual (PDM): rationale, conception, and structure. J Am Psychoanal Assoc 2011, 59(1):153-64.

20. Engel GL: The need for a new medical model: a challenge for biomedicine. Science 1977, 196(4286):129-36.

21. Ghaemi N: The concepts of psychiatry: A pluralistic approach to the mind and mental illness. Baltimore: Johns Hopkins University Press; 2003.

22. Eid M: The Bio-Psycho-Social model: how accurate and valid is it? J Psychol Psychother. 2012, 2:e103.

23. Del Puente G, Bragazzi NL: The Bio-psycho-social model and beyond: its limitations and the need for a new model. A response to Eid's Editorial, "The Bio-Psycho-Social model: how accurate and valid is it? Open Access Scientific Reports. 2012, 1:399.

24. Andrews G, Goldberg DP, Krueger RF, Carpenter WT, Hyman SE, Sachdev P, Pine DS: Exploring the feasibility of a meta-structure for DSM-V and ICD-11: could it improve utility and validity? Psychol Med 2009, 39(12):1993-2000.

25. Gottesman II, Gould TD: The endophenotype concept in psychiatry: etymology and strategic intentions. Am J Psychiatry 2003, 160(4):636-45.

26. Giordano J: Neuroimaging in psychiatry: approaching the puzzle as a piece of the bigger picture(s). AJOB-Neuroscience 2012, 3(4):54-56.

27. Giordano J, Engebretson J, Benedikter R: Pain and culture: considerations for meaning and context. Cambridge Quarterly Rev. Healthcare Ethics. 2008 77:45-59.

28. Giordano J, Wurzman R: Neurological disease and depression: the possibility and plausibility of putative neuropsychiatric spectrum disorders. Depression: Mind and Body 2008, 4(1):2-5.

29. Ozdemir V, Suarez-Kurtz G, Stenne R, Somogyi AA, Someya T, Kayaalp SO, Kolker E: Risk assessment and communication tools for genotype associations with multifactorial phenotypes: the concept of "edge effect" and cultivating an ethical bridge between omics innovations and society. OMICS 2009, 13(1):43-61.

30. Goh KI, Cusick ME, Valle D, Childs B, Vidal M, Barabási AL: The human disease network (the human diseasome). Proc Natl Acad Sci USA 2007, 104:8685-8690.

31. Alam S, Patel J, Giordano J: Working towards a new psychiatry - neuroscience, technology and the DSM-5. Philos Ethics Humanit Med 2012, 7:1.

32. Giordano J, Engebretson JC: Don't ignore religious beliefs. Tex Med 2005, 101(2):7-8.

33. Richerson PJ, Boyd R, Henrich J: Gene-culture coevolution in the age of genomics. Proc Natl Acad Sci U S A 2010, 107(Suppl 2):8985-92.

34. Wurzman R, Giordano J: Differential susceptibility to plasticity: a 'missing link' between gene culture co-evolution and neuropsychiatric spectrum disorders? BMC Med 2012, 10:37.

35. Belsky J, Jonassaint C, Pluess M, Stanton M, Brummett B, Williams R: Vulnerability genes or plasticity genes? Mol Psychiatry 2009, 14:746-754.

36. Caspi A, Moffitt TE: Gene-environment interactions in psychiatry: Joining forces with neuroscience? Nat Rev Neurosci 2006, 7:583-590.

37. Costae Silva JA: Personalized medicine in psychiatry: new technologies and approaches. Metabolism 2013, 62:1-40.

38. Turck CW, Maccarrone G, Sayan-Ayata E, Jacob AM, Ditzen C, Kronsbein H, Birg I, Doertbudak CC, Haegler K, Lebar M, Teplytska L, Kolb N, Uwaje N, Zollinger R: The quest for brain disorder biomarkers. J Med Invest 2005, 52 Suppl:231-5.

39. Bragazzi NL, Sivozhelezov V, Nicolini C: Leader gene: a fast data-mining tool for molecular genomics. J Proteomics Bioinform 2011, 4(4):083-086.

40. Racapé M, Bragazzi N, Sivozhelezov V, Danger R, Pechkova E, Nicolini C, Brouard S, Soulillou JP, Duong Van Huyen JP: SMILE silencing and PMA activation gene networks in HeLa cells: comparison with kidney transplantation gene networks. J Cell Biochem 2012, 113(6):1820-32.

41. Orlando B, Bragazzi N, Nicolini C: Bioinformatics and systems biology analysis of genes network involved in OLP (Oral Lichen Planus) pathogenesis. Arch Oral Biol 2013, 58(6):664-73.

42. Nicolini C, Bragazzi N, Pechkova E: Nanoproteomics enabling personalized nanomedicine. Adv Drug Deliv Rev 2012, 64(13):1522-31.

43. Nicolini C, Bruzzese D, Cambria MT, Bragazzi NL, Pechkova E: Recombinant laccase: I Enzyme cloning and characterization. J Cell Biochem 2013, 114(3):599-605.
44. Bragazzi NL, Pechkova E, Scudieri D, Terencio TB, Adami M, Nicolini C: Recombinant laccase: II. Medical biosensor. Crit Rev Eukaryot Gene Expr 2012, 22(3):197-203.

45. Nicolini C, Adami M, Sartore M, Bragazzi NL, Bavastrello V, Spera R, Pechkova E: Prototypes of newly conceived inorganic and biological sensors for health and environmental applications. Sensors (Basel) 2012, 12(12):17112-27.

46. Bragazzi NL, Del Puente G: Panic attacks and possession by djinns: lessons from ethnopsychiatry. Psychol Res Behav Manag 2012, 5:185-90.

doi:10.1186/1747-5341-8-4

Cite this article as: Bragazzi: Rethinking psychiatry with OMICS science in the age of personalized P5 medicine: ready for psychiatome?

Philosophy, Ethics, and Humanities in Medicine 2013 8:4.

\section{Submit your next manuscript to BioMed Central and take full advantage of:}

- Convenient online submission

- Thorough peer review

- No space constraints or color figure charges

- Immediate publication on acceptance

- Inclusion in PubMed, CAS, Scopus and Google Scholar

- Research which is freely available for redistribution 